\title{
Molecular and Structural Preservation of Dehydrated Bio-Tissue for $\mathrm{THz}$ Spectroscopy
}

\author{
Gretel M. Png ${ }^{a}$, Jin Wook Choi ${ }^{b}$, Ian Guest ${ }^{c}$, Brian W.-H. Ng${ }^{a}$, Samuel P. Mickan ${ }^{a}$, Derek \\ Abbott $^{a}$, Xi-Cheng Zhang ${ }^{b}$ \\ ${ }^{a}$ Centre for Biomedical Engineering, School of Electrical and Electronic Engineering, The \\ University of Adelaide, SA 5005, Australia; \\ ${ }^{b}$ Center for Terahertz Research, Rensselaer Polytechnic Institute, Troy, NY 12180, USA; \\ ${ }^{c}$ Wadsworth Center, NYS Department of Health, Empire State Plaza, Albany, NY 12201, USA
}

\begin{abstract}
Terahertz transmission through freshly excised biological tissue is limited by the tissue's high water content. Tissue fixation methods that remove water, such as fixation in Formalin, destroy the structural information of proteins hence are not suitable for $\mathrm{THz}$ applications. Dehydration is one possible method for revealing the tissue's underlying molecular structure and components. In this study, we measured the THz responses over time of dehydrating fresh, necrotic and lyophilized rat tissue. Our results show that as expected, THz absorption increases dramatically with drying and tissue freshness can be maintained through lyophilization. Dehydrated biological tissue with retained molecular structure can be useful for future laser-based $\mathrm{THz}$ wave molecular analysis.
\end{abstract}

Keywords: terahertz, T-rays, bio-tissue, dehydration, absorption

\section{INTRODUCTION}

Coherent spectroscopic information at terahertz $\left(1 \mathrm{THz}=10^{12} \mathrm{~Hz}\right)$ frequencies may allow us to study the health of human tissue and diagnose diseases that have until now been undetectable by other imaging modalities. The human body however contains around $60 \%$ water ${ }^{1}$ so both in situ and excised tissue contain high amounts of water that attenuate the strength of transmitted THz significantly. ${ }^{2}$ Studies of excised human and animal tissue that have been performed previously ${ }^{3-10}$ reveal that the transmitted $\mathrm{THz}$ signals from freshly excised tissue are often noisy and weak as expected due to high tissue hydration. Two pieces of information that are often lacking in existing literature are the physical condition of the tissue during measurements, and how the tissue is preserved prior to measurements. The former is important because excised tissue does not remain moist indefinitely. In a nitrogen-purged THz-Time Domain System (THz-TDS), tissue drying is accelerated. As water evaporates, the tissue also becomes thinner. The measured $\mathrm{THz}$ signal therefore increases over time and the tissue's thickness needs to be monitored in order to accurately calculate its optical properties. Tissue preservation is also an issue because some preservation methods can damage the very molecular structures that interact with $\mathrm{THz}$ to produce the unique spectroscopic information.

This paper presents a study of the effects of dehydration and preservation on the THz responses of three types of rat tissue. A brief introduction to current tissue preservation methods will be provided, then the results of tissue dehydration will be presented. The effects of tissue preservation will follow, and finally recommendations for future work are made.

Further author information: (Send correspondence to Gretel Png)

E-mail: gpng@eleceng.adelaide.edu.au, Telephone: +61 (0)8 83036296

E-mail: bwng@eleceng.adelaide.edu.au, Telephone: +61 (0)8 83035054

E-mail: dabbott@eleceng.adelaide.edu.au, Telephone: +61 (0)8 83035748

Biomedical Applications of Micro- and Nanoengineering III, edited by Dan V. Nicolau, Proc. of SPIE Vol. 6416, 64160W, (2006) · 1605-7422/06/\$15 · doi: 10.1117/12.695767 


\section{POST-BIOPSY TISSUE PRESERVATION FOR PATHOLOGICAL ANALYSIS}

This section aims to highlight existing tissue preservation methods used in biopsies. Biopsies are of interest because the small quantity of cells and/or tissue removed from a patient is in theory sufficient for $\mathrm{THz}$ spectroscopy. There are several ways of performing a biopsy and the appropriate method is chosen depending on the type and location of the tissue in the body. For example, punch biopsy is one common method for sampling rashes and masses on the skin: a cylindrical piece of skin with 3 or $4 \mathrm{~mm}$ diameter is sampled and the wound is closed via sutures. For masses deeper in the skin, a simple core needle is used: two or more cylindrical cores of tissue with $2 \mathrm{~mm}$ diameter and $10 \mathrm{~mm}$ length are extracted - the multiple cores increase the diagnostic accuracy. For tissue deep inside the body, a Fine Needle Aspiration Biopsy (FNAB) may be used to extract cells. Detailed descriptions of biopsies can be found in References 11 and 12. When a tissue section is removed, it needs to be fixed and sliced because the pathological examination requires viewing the tissue slice under an optical microscope. Formalin fixing is one common method that provides mechanical support for slicing through denaturation of proteins, but since protein structure preservation is important in $\mathrm{THz}$ bio-related analysis, Formalin fixing is unsuitable.

Tissue sections can also be frozen and then sliced. Domestic freezers are not suitable for freezing tissues used in pathological examination because the slow freezing process causes the formation of ice crystals in the tissue and may damage it. Quick-freezing (or rapid-freezing) is used instead. ${ }^{13}$ The tissue section is dropped into a refrigerant such as liquid nitrogen, isopentane or isobutane, and freezes immediately at around $-80^{\circ} \mathrm{C}$. The frozen tissue can then be sliced by a microtome* housed inside a cryostat. Most microtomes can slice tissue to thicknesses up to $50 \mu \mathrm{m}$ - the same order of wavelength in the optical range $\left(\lambda=10^{-6} \mathrm{~m}\right)$ but three orders less than in the $\mathrm{THz}$ range $\left(\lambda=10^{-3} \mathrm{~m}\right)$, thus creating a diffraction limited situation. Quick-frozen tissue sections should therefore be sliced into thicker sections (in the order of $10^{-3} \mathrm{~m}$ ) for $\mathrm{THz}$ applications.

Another possible tissue preservation method is dehydration. It is not used in biopsies but is used in a process called lyophilization (freeze-drying). A tissue section is quick-frozen as described above and is then placed in a low-pressure (or high-vacuum, 10-100 Pa), low-temperature (approximately $-80^{\circ} \mathrm{C}$ ) environment that causes water to sublimate (change directly from the solid/ice state to the gaseous state). This process removes $>90 \%$ of water in the tissue whilst retaining its structural and molecular integrity. Freeze-dried coffee beans are an example of how freshness is preserved in food. The lyophilizer used in this study is shown in Figure 1(a). A section of ventral muscle in its fresh and lyophilized forms are shown in Figure 1(b); water is removed from the lyophilized tissue without visible damage to its physical structure. Tissues preserved through lyophilization are therefore good candidates for $\mathrm{THz}$ spectroscopy.

If tissue is not preserved, then necrosis (and eventually decomposition) will occur. In necrosis, normal molecular structure is lost thus reducing the richness of $\mathrm{THz}$ spectroscopic information from a tissue section.

\section{SAMPLE PREPARATION}

Tissue sections used in this study were obtained from the Wadsworth Medical Center in Albany, New York, USA. One white rat was sacrificed for a routine autopsy unrelated to this study. Various organs were excised after the autopsy. In order to preserve the freshness of the excised organs, they were submerged in a Petri dish containing Hank's buffer. Hank's buffer is a glucose-enriched buffer that can sustain the organs for a limited period. The Petri dish was seated on a bed of ice to keep the buffer cold.

Each organ was sliced into three or more sections ("slices"). Multiple slices were required so that one slice could be kept fresh in Hank's buffer, one slice could be lyophilized, and one slice could be placed in a buffer with no glucose to induce necrosis; an example of such a buffer is the Phosphate-Based Solution (PBS). As highlighted in Section 2, the thickness of the tissue slices should be in the order of $10^{-3} \mathrm{~m}$. The small size of rat organs and their pliability, make them difficult to handle and slice. Several custom made slicing jigs were tested, ranging from simple industrial-grade razor blades, to commercially purchased tissue matrices, such as the brain matrix from Braintree Scientific Inc. ${ }^{14}$ A vibratome ${ }^{\dagger}$ was also tested and could achieve thicknesses of $500 \mu \mathrm{m}$

\footnotetext{
${ }^{*}$ A microtome holds a blade that is used to slice tissue with a cutting action analogous to cutting thin slices of meat on a cutting board.

${ }^{\dagger}$ In a vibratome, the blade vibrates to achieve the cutting action.
} 


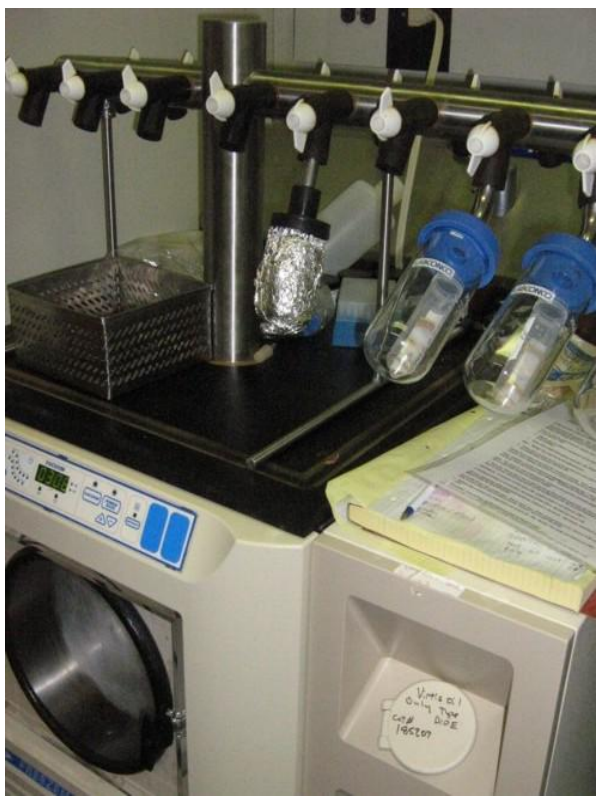

(a) Lyophilizer used in this study.
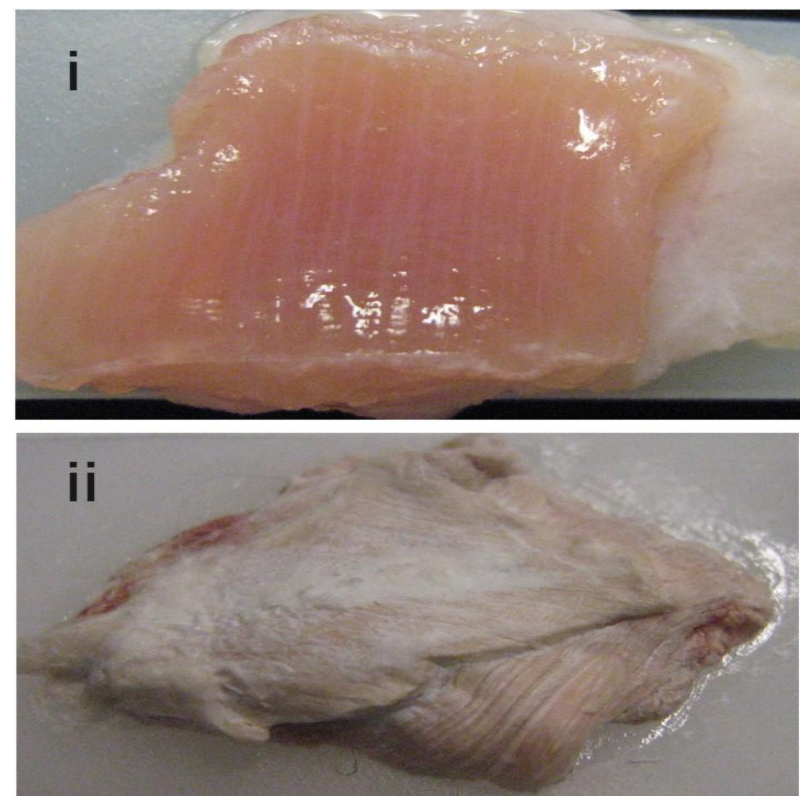

(b) (i) Fresh and (ii) lyophilized ventral muscle sections.

Figure 1. (a) The operating temperature of the lyophilizer was preset to $-73.5^{\circ} \mathrm{C}$ and the pressure set to $212 \mathrm{mTorr}(\sim 28$ $\mathrm{Pa}$ ). To remove $>90 \%$ water in this particular machine, tissues placed in the large glass vials with blue airtight seals were lyophilized for at least 24 hours; (b) Despite the removal of $>90 \%$ of water, the lyophilized rat ventral muscle section keeps its shape and thickness. The only visible differences are the color, texture, and most significantly, the firmness that allows easier handling of the tissue when compared to supple fresh tissue.

and $1000 \mu \mathrm{m}$, but it took more than an hour to complete one slice, and thus was deemed unfeasible. It was found that 2 razor blades, spaced apart by $1 \mathrm{~mm}$ with nuts and washers, could produce a fairly good cut. The brain matrix produced the best cut. The organs were sliced immediately after excision and if possible, sliced whilst submerged in Hank's buffer. Otherwise, the slices were submerged in Hank's buffer as soon as possible after slicing.

The slices were mounted on $1 \mathrm{~mm}$ polyethylene plates (Goodfellow, high density sheet polyethylene, 0.95 $\left.\mathrm{g} / \mathrm{cm}^{3}\right)$. Each plate was about $60 \mathrm{~mm}$ by $20 \mathrm{~mm}$ in size to fit into the existing sample holder. The slices were placed gently on the plates without any visible stretching of the tissue, and secured at the edges by minimal drops of commercial superglue ${ }^{\ddagger}$. The slices were then divided into 3 batches: one batch was re-submerged in Hank's buffer ("fresh tissue"), one batch was submerged in a Petri dish of PBS ("necrotic tissue"). The last batch was instantly frozen in a beaker of isobutane, which was seated in dry ice to keep the isobutane at $\sim-60^{\circ} \mathrm{C}$. The tissue were then transferred into the lyophilizer shown in Figure 1(a) and lyophilized for 48 hours ("lyophilized tissue"). The Petri dishes containing the fresh and necrotic tissue, were packed in a styrofoam box together with ice packs, and transported for 20 minutes to the THz laboratory. The lyophilized tissue were transported two days later in test tubes that were sealed at the mouth with Parafilm ${ }^{\circledR} \mathrm{M}$.

\section{TEST AND RESULTS}

The THz system used in this study was a standard THz-Time Domain System (TDS) based on a 100 fs, 800 nm Ti:Sapphire laser (Coherent Mira $\left.{ }^{\mathrm{TM}} 900\right)$. THz was emitted and detected using optical rectification in ZnTe crystals. Details of a THz-TDS system can be found in Reference 15. Dry nitrogen gas was piped into the test chamber, where the humidity was kept below $3 \% \pm 3 \%$ and the temperature was close to ambient $\left(22-23^{\circ} \mathrm{C}\right)$.

\footnotetext{
${ }^{\ddagger}$ From private correspondence with technical staff at the Wadsworth Center, this is a common method for securing tissue.
} 
The necrotic tissue were transferred into a domestic refrigerator and allowed to sit for a further 24 hours. The fresh tissue slices remained in the styrofoam box and were taken out individually from the Hank's buffer just prior to each $\mathrm{THz}$ measurement. The ice packs were replaced when necessary. Each fresh slice was therefore fresh up until the moment of measurement. Excess Hank's buffer was allowed to drip off the tissue by tilting the polyethylene plate. The plate was then mounted vertically in the $\mathrm{THz}$ test chamber with the tissue at the $\mathrm{THz}$ focal point. Five or more measurements of each slice of tissue were then made.

\subsection{Effects of Dehydration on Tissue THz Response}

It was found that in the first run of measurements, the tissue were often so moist that the measured $\mathrm{THz}$ signal resembled that of noise. Figure 2(a) presents the first liver measurement plotted together with the $\mathrm{THz}$ noise signal - the liver's THz signal is barely above the noise floor (-21 dB on average). This has been similarly observed in an earlier study of tissue. ${ }^{10}$

Existing literature often lacks information on how long tissue samples are exposed to the dry test environment. This is an important issue because water in tissue will dehydrate over time, and the rate of evaporation is accelerated for thin tissue slices in a nitrogen-purged environment. The results reported by ${ }^{8-10}$ for human and animal tissue show that the usable $\mathrm{THz}$ bandwidth is narrow, typically between 0.5 to $1.5 \mathrm{THz}$. This is expected because the high tissue water content absorbs higher $\mathrm{THz}$ frequencies. When left to dry however, the reduction in tissue water content will result in lower absorption hence an increase in the usable bandwidth. In this study, each tissue slice was left in the nitrogen-purged test chamber and its $\mathrm{THz}$ time domain signal measured every 5 to 10 minutes; the time instance of every measurement was recorded.

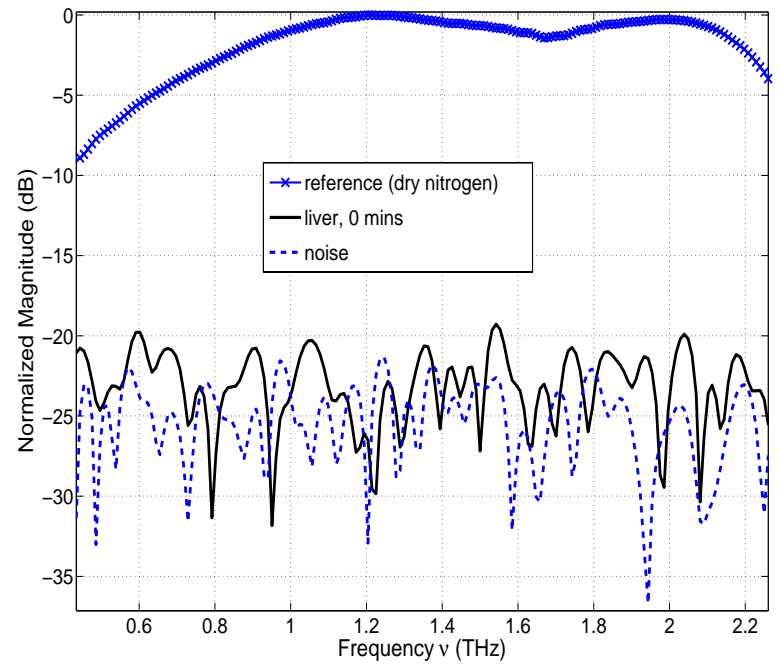

(a) A comparison of the $\mathrm{THz}$ frequency response of liver and noise.

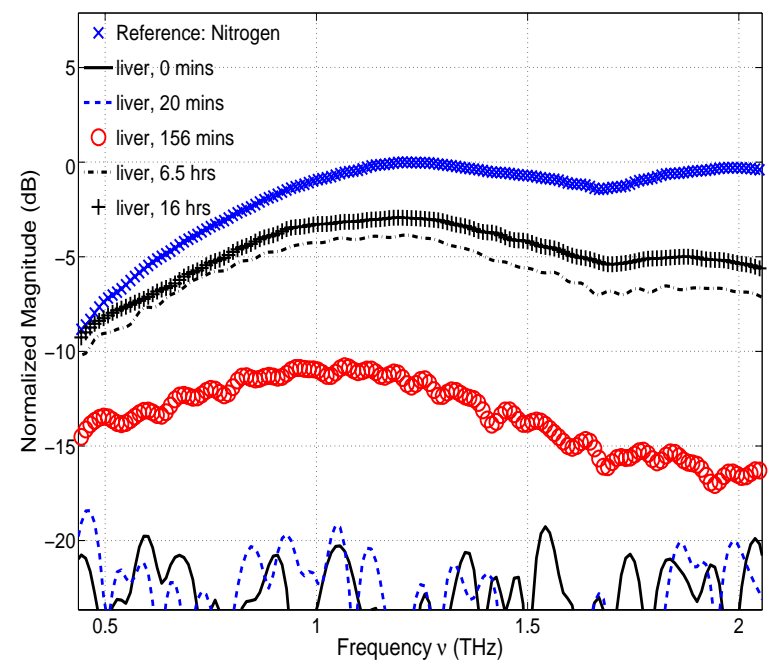

(b) $\mathrm{THz}$ frequency response of liver over time.

Figure 2. (a) The signal to noise ratio of a very moist slice of liver is comparable to that of the system noise. The noise floor of the system used in this study is $-21 \mathrm{~dB}$; (b) As water in the slice of liver evaporates, the signal to noise ratio increases above the noise floor of $-21 \mathrm{~dB}$. The time taken to dehydrate liver such that an increase in bandwidth is visible on this plot was only about 20 minutes.

The increase in the usable $\mathrm{THz}$ bandwidth over time for liver is shown in Figure 2(b). The usable bandwidth above the noise floor for the first two measurements is narrow but widens as the tissue dehydrates. To emphasize this change, the frequency response after 156 minutes is plotted to highlight the broadening of the usable bandwidth to beyond $2 \mathrm{THz}$.

The increase in the bandwidth was also observed in other tissue types. A comparison of the plots in Figures 2(b), 3(a) and 3(b) shows that all three samples contain high amounts of water initially, but there is a difference 
in the time it takes for the measured $\mathrm{THz}$ signal to rise above the noise floor. The dissimilar rate of dehydration may be due to different tissue densities but further investigation is required.

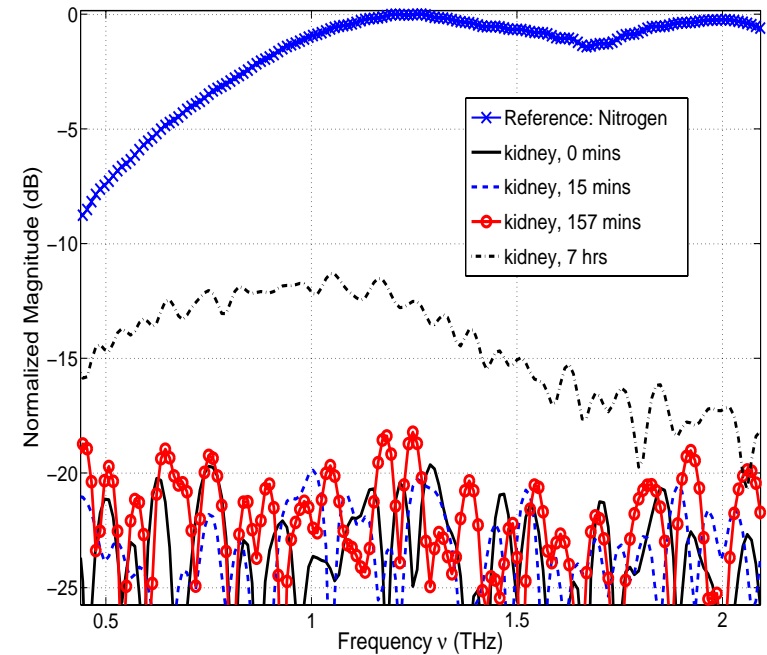

(a) $\mathrm{THz}$ frequency response of kidney over time.

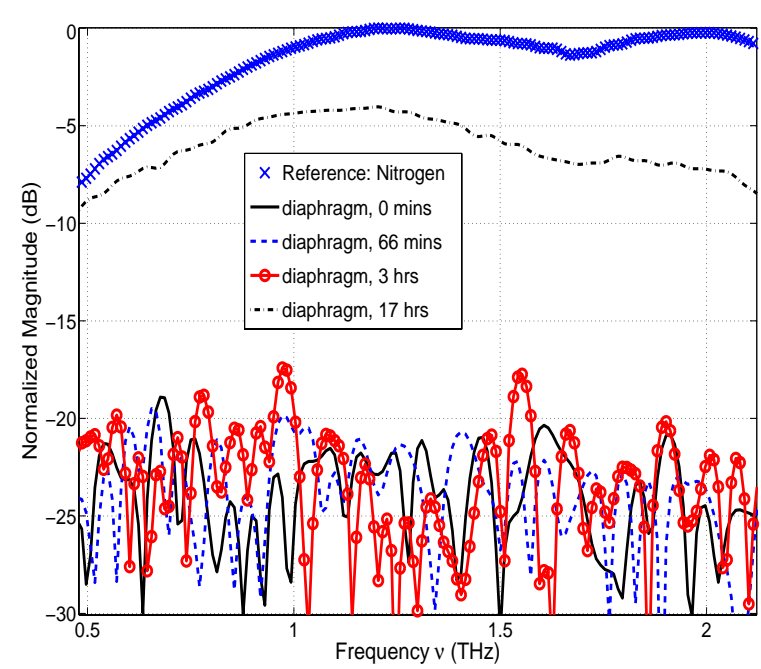

(b) $\mathrm{THz}$ frequency response of diaphragm over time.

Figure 3. A comparison of the frequency response of liver in Figure 2(b) with that of kidney and diaphragm shows that: (a) after 7 hours, the signal to noise ratio of kidney was $-13 \mathrm{~dB}$, but the signal to noise ratio of liver was $-4 \mathrm{~dB}$, whereas (b) it took 17 hours for the signal to noise ratio of diaphragm to rise to $-4 \mathrm{~dB}$.

With the knowledge of sample thickness $d$, incident $\mathrm{THz}$ signal $E_{\text {in }}$, and transmitted $\mathrm{THz}$ signal $E_{\text {out }}$, the absorption coefficient $\alpha$ can be calculated based on the Beer-Lambert law:

$$
\alpha=\frac{-\ln \left[\left(\frac{E_{\text {out }}}{E_{\text {in }}}\right)^{2}\right]}{d}
$$

One problem encountered with dehydration is that the thickness of the tissue slice decreases over time. This means the thickness of the slices must be measured regularly because Equation 1 shows that the absorption coefficient is proportional to the reciprocal of thickness $d$. Figure 4 shows the changes in absorption over time due to tissue dehydration.

There is a prominent oscillatory artifact in the early measurements of all tissue which is possibly due to the initial presence of large amounts of water. Some of the peaks appear to be in the proximity of the water lines reported in Reference 2 (namely $0.989,1.209,1.23$ and $1.412 \mathrm{THz}$ ), but it is not possible to tell at this stage if these peaks are unique "bio-tissue water lines". Future investigation will look into the interaction of tissue water with $\mathrm{THz}$.

\subsection{Sensing Necrosis in Rat Tissue Using THz}

In this study, the dehydration process was conducted over 24 hours or more at ambient temperature. The question of whether the dehydrated tissue is still considered "fresh" is a valid one. The batch of tissue that were submerged in PBS and left in the domestic refrigerator ("necrotic tissue" - cf. Section 3) were measured and compared against the fresh tissue. A visual inspection of the necrotic tissue while submerged in PBS revealed some discoloration but other visual cues where not convincing. The stench, however, was overpowering. Like the fresh tissue, early measurements of the necrotic tissue suffered from high water absorption. As water evaporated, the THz signal improved. Figure 5(a) presents the absorption coefficient of necrotic tissue alongside fresh tissue. Since protein denaturation occurs in necrosis, it is expected that less protein- $\mathrm{THz}$ interaction occurs, hence more $\mathrm{THz}$ will transmit through a necrotic sample without absorption. In Figure 5(a), necrotic liver exhibits lower 


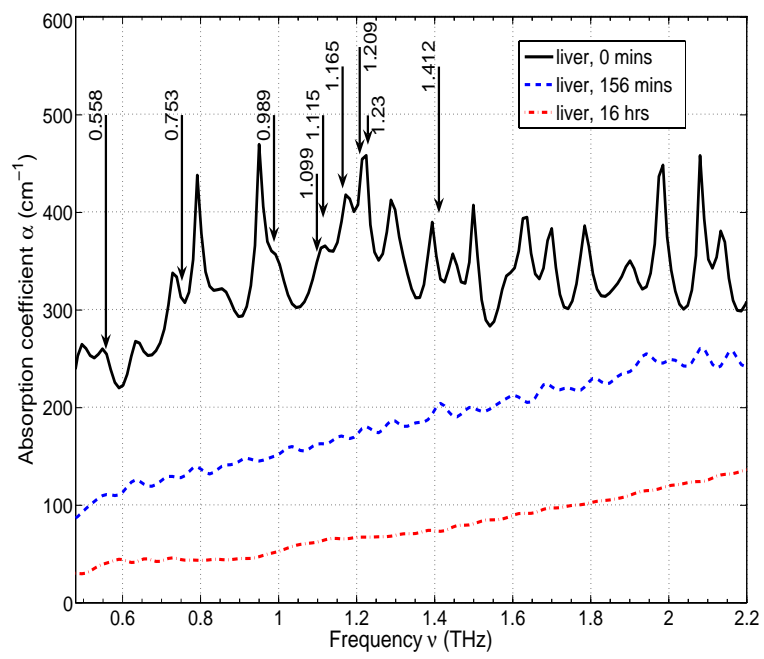

(a) Changes in the absorption profile of fresh rat liver over time.

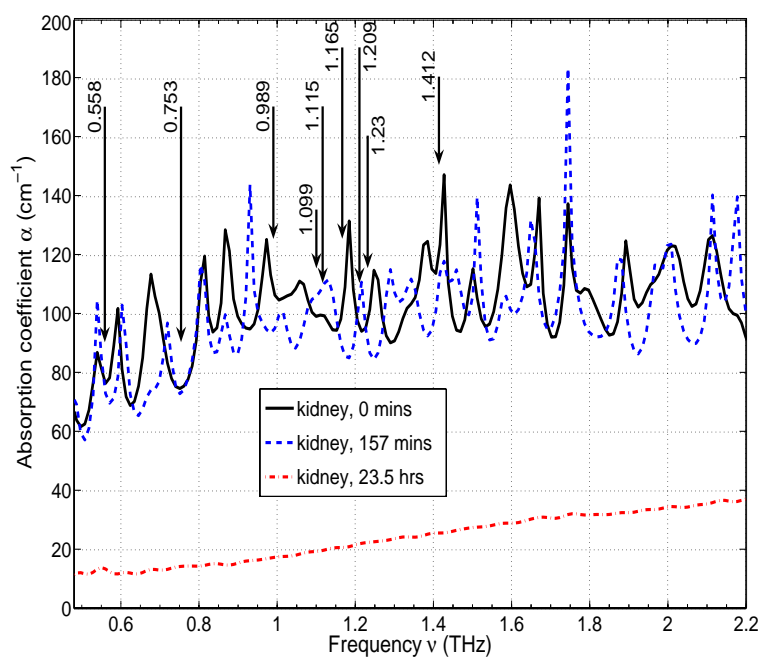

(b) Changes in the absorption profile of fresh rat kidney over time.

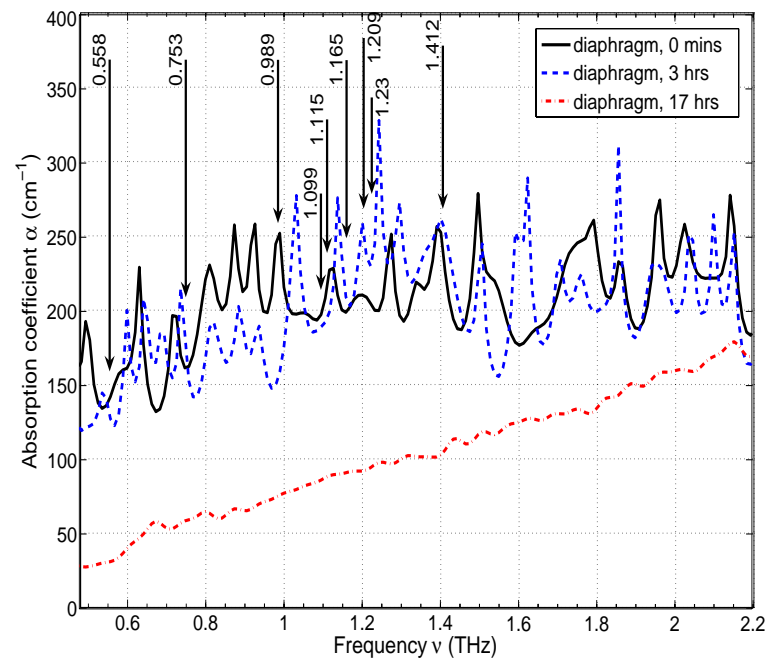

(c) Changes in the absorption profile of fresh rat diaphragm over time.

Figure 4. Absorption profiles of fresh rat tissue over time with known water lines from Reference 2 juxtaposed. Peaks in the early measurements of all three tissue types appear to either correspond or are in the proximity of water lines 0.989 , 1.209, 1.23 and $1.412 \mathrm{THz}$. As water evaporates, the plots become more linear and the absorption reduces as expected.

$\mathrm{THz}$ absorption for the same duration of drying time as fresh liver. This is similarly observed for the other tissue types. The answer to the question of freshness based on $\mathrm{THz}$ response is that it is very likely that protein denaturation has not occurred in dehydrated fresh tissue because there is no visible correlation between the absorption profiles of fresh and necrotic tissue.

\subsection{Comparison of Lyophilized and Dehydrated Tissue}

As highlighted in Section 2, lyophilization is a preservation method that ensures the molecular composition and structure of tissue is maintained whilst $90 \%$ of water is removed. An optical image of a piece of lyophilized tissue is shown in Figure 5(b) (image iii). Although more water can be removed from a tissue by extending the lyophilization time, the tissue becomes brittle and cracks easily. The sample shown in Figure 5(b) had small 


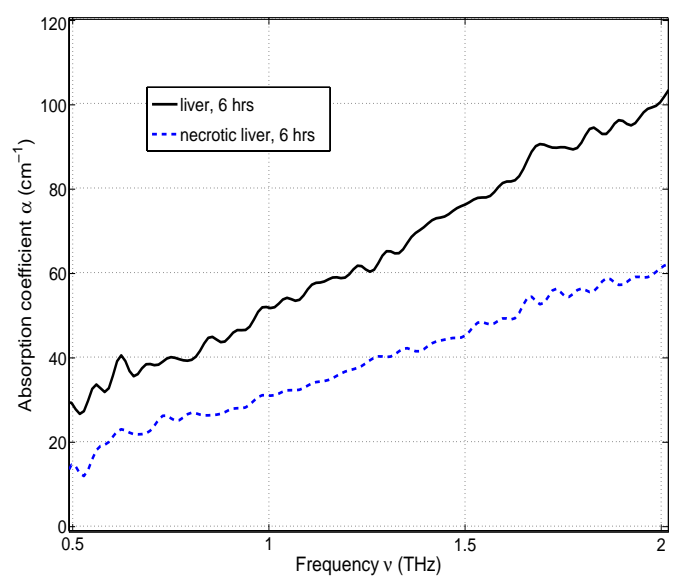

(a) Calculated absorption coefficients of fresh and necrotic rat liver.
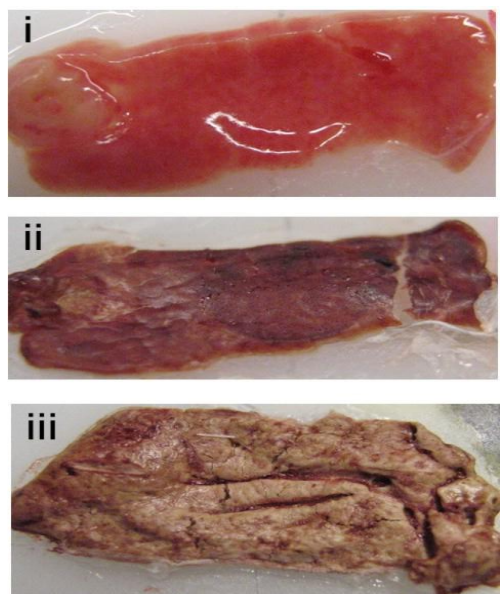

(b) Optical images of (i) fresh rat liver, (ii) dehydrated fresh rat liver, and (iii) lyophilized rat liver.

Figure 5. (a) The absorption coefficient of necrotic liver is lower than that of fresh liver. This is consistent with the notion that protein denaturation occurs during necrosis hence less protein- $\mathrm{THz}$ interaction occurs, resulting in a $\mathrm{THz}$ response that resembles that of a non-absorbing medium in the $\mathrm{THz}$ regime; (b) The visible signs of dehydration can be seen (shrinkage, thinning) in image ii. Wrinkling and tearing can also happen during dehydration, although this sample only suffered tearing in an area that was not in the $\mathrm{THz}$ beam path. Image iii shows a different fresh liver sample after lyophilization.

cracks, and during measurements had to be positioned such that the $\mathrm{THz}$ beam would not pass through the cracks .

Figure 6 presents the absorption profile of lyophilized tissue and fresh tissue that have been dehydrated for 12 hours or more. All three lyophilized samples shown in Figure 6 have lower absorption than the fresh samples. It is noted that the slopes of the lyophilized liver and kidney plots are similar to those of fresh liver and kidney, but the slopes of the diaphragm plots differ. One possibility is that the physical condition of the samples (e.g., cracks) may be the cause.

\section{CONCLUSION AND FUTURE WORK}

This paper has presented results from a study on how dehydration affects the $\mathrm{THz}$ response of excised rat tissue. Several tissue preservation methods were introduced and a description was provided on how tissue in this study were prepared for $\mathrm{THz}$ measurements. In addition to fresh tissue, lyophilized and necrotic tissue were investigated for the first time with $\mathrm{THz}$ and compared against fresh tissue. The results have shown that, as expected, the high water content in fresh tissue strongly absorbs $\mathrm{THz}$ but if the tissue is left to dry, the absorption reduces. To check if the process of dehydrating fresh tissue causes necrosis, the $\mathrm{THz}$ absorption profiles of fresh and necrotic tissue were compared, and the result indicates that necrotic tissue has lower $\mathrm{THz}$ absorption, possibly due to protein denaturation. Since lyophilization preserves tissue molecular and structural information, the THz response from lyophilized tissue may reveal molecular information previously unseen in fresh tissue due to masking by water. Structural and molecular $\mathrm{THz}$ studies of lyophilized tissue and bio-materials will be the focus of future work.

\section{ACKNOWLEDGMENTS}

We wish to thank the University of Adelaide, the Australian Research Council, the Cooperative Research Centre for Sensor Signal and Information Processing for financial support. The author also wishes to thank the SPIE, the University of Adelaide Alumni, and the AUGU/RC Heddle Award Committee for travel support to Rensselaer Polytechnic Institute. 


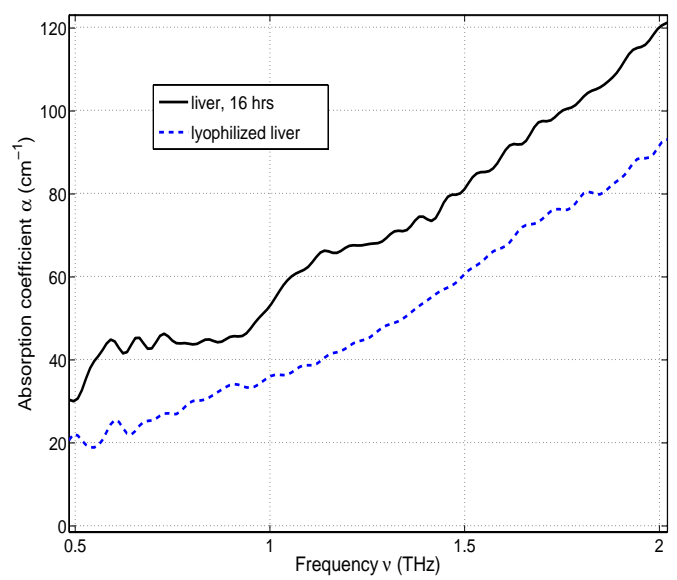

(a) Calculated absorption coefficients of lyophilized and fresh rat liver.

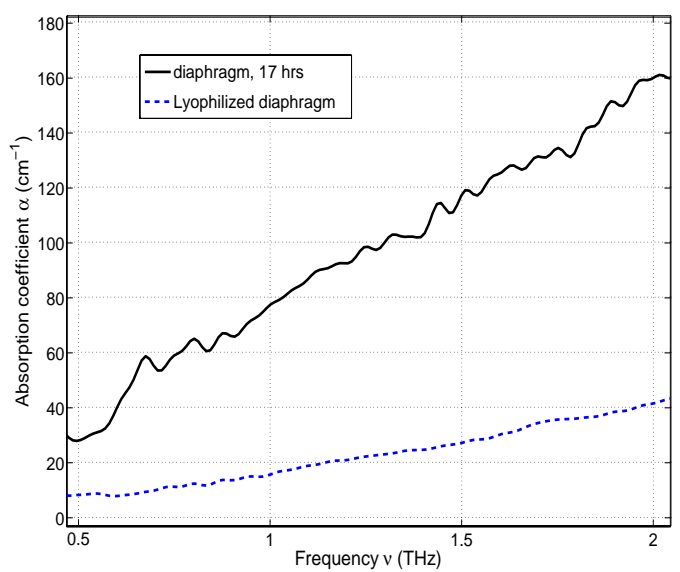

(c) Calculated absorption coefficients of lyophilized and fresh rat diaphragm.

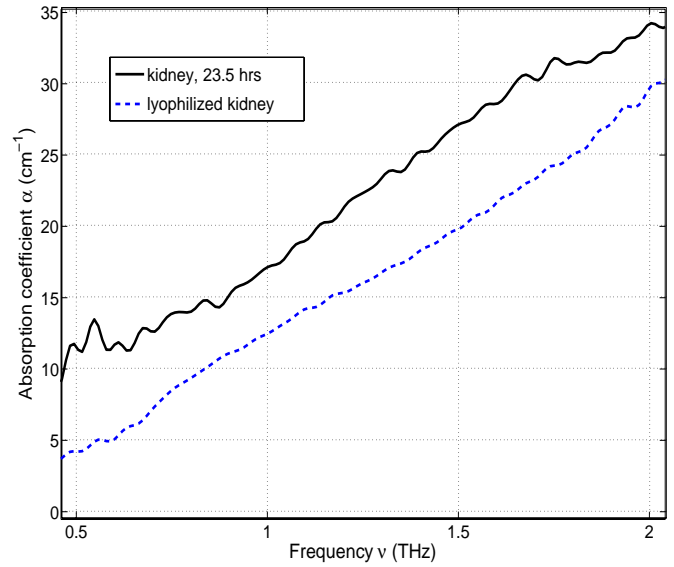

(b) Calculated absorption coefficients of lyophilized and fresh rat kidney.
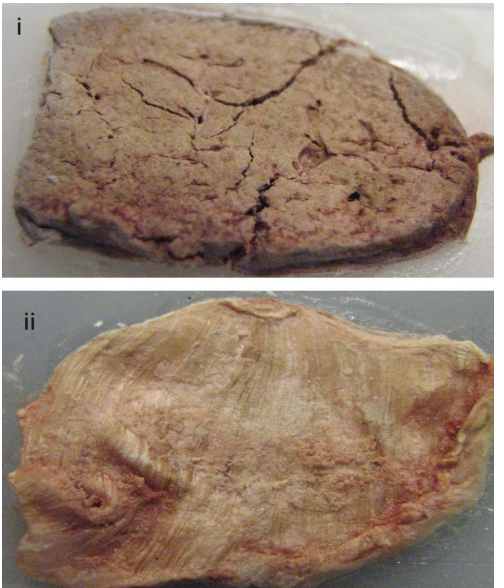

(d) Optical images of (i) lyophilized rat kidney, (ii) lyophilized rat diaphragm.

Figure 6. (a-c) For all samples, the THz absorption in lyophilized tissue is lower than that of fresh tissue.

\section{REFERENCES}

1. R. Mullins, Fluid, Electrolytes, and Shock. Sabiston Textbook of Surgery: The Biological Basis of Modern Surgical Practices, W.B. Saunders Company, Philadelphia, PA, USA, 16th ed., 2001.

2. M. van Exter, C. Fattinger, and D. Grischkowsky, "Terahertz time-domain spectroscopy of water-vapor," Optics Letters 14(20), pp. 1128-1130, 1989.

3. P. Knobloch, C. Schildknecht, T. Kleine-Ostmann, M. Koch, S. Hoffmann, M. Hofmann, E. Rehberg, M. Sperling, K. Donhuijsen, G. Hein, and K. Pierz, "Medical THz imaging: an investigation of histopathological samples," Physics in Medicine and Biology 47, pp. 3875-3884, 2002.

4. B. Ferguson, S. Wang, D. Gray, D. Abbott, and X.-C. Zhang, "Identification of biological tissue using chirped probe THz imaging," Microelectronics Journal 33(12), pp. 1043-1051, 2002.

5. R. Woodward, B. Cole, V. Wallace, R. Pye, D. Arnone, E. Linfield, and M. Pepper, "Terahertz pulse imaging in reflection geometry of human skin cancer and skin tissue," Physics in Medicine and Biology 47(21), pp. 3853-3863, 2002.

6. R. Woodward, V. Wallace, R. Pye, B. Cole, D. Arnone, E. Linfield, and M. Pepper, "Terahertz pulse imaging of ex-vivo Basal Cell Carcinoma," Journal of Investigative Dermatology 120(1), pp. 72-78, 2003. 
7. R. Woodward, V. Wallace, D. Arnone, E. Linfield, and M. Pepper, "Terahertz pulsed imaging of skin cancer in the time and frequency domain," Journal of Biological Physics 29(2-3), pp. 257-261, 2003.

8. A. Fitzgerald, E. Berry, N. Zinov'ev, S. Homer-Vanniasinkam, R. Miles, J. Chamberlain, and M. Smith, "Catalogue of human tissue optical properties at terahertz frequencies," Journal of Biological Physics 129(23), pp. 123-128, 2003.

9. E. Berry, A. Fitzgerald, N. Zinov'ev, G. Walker, S. Homer-Vanniasinkam, C. Sudworth, R. Miles, J. Chamberlain, and M. Smith, "Optical properties of tissue measured using terahertz-pulsed imaging," in Proc. SPIE Medical Imaging 2003: Physics of Medical Imaging, M. Yaffe and L. Antonuk, eds., 5030, pp. 459470, (San Diego, CA, USA), 2003.

10. M. He, A. Azad, S. Ye, and W. Zhang, "Far-infrared signature of animal tissues characterized by terahertz time-domain spectroscopy," Optics Communications 259(1), pp. 389-392, 2006.

11. C. Townsend Jr., R. Beauchamp, B. Evers, and K. Mattox, Sabiston Textbook of Surgery: The Biological Basis of Modern Surgical Practices, W.B. Saunders Company, Philadelphia, PA, USA, 16th ed., 2001.

12. L. Ackerman, J. del Regato, H. Spjut, and J. Cox, Ackerman and Del Regato's Cancer: Diagnosis, Treatment, and Prognosis, C.V. Mosby, St. Louis, MO, USA, 6th ed., 1984.

13. A.-Y. Leong and G.-W. Leong, Cancer Explained: Causes, Diagnosis, Treatment and Prevention, P G Publishing, Singapore, 1989.

14. "Brain matrices," <http://www.braintreesci.com/matrice.htm\#Acrylic>, (Accessed: 2006-12-01).

15. P. Siegel, "Terahertz technology," IEEE Transactions on Microwave Theory and Techniques 50(3), pp. 910928, 2002. 